\title{
Reduction Effect of Calcium Alginate on Blood Triglyceride Levels Causing the Inhibition of Hepatic and Total Body Accumulation of Fat in Rats
}

\author{
Fumiyoshi Kasahara, ${ }^{a, b}$ Takako Kato, ${ }^{a, c}$ Yoko Idota, ${ }^{a, c}$ Hitoe Takahashi, ${ }^{a, c}$ Chihaya Kakinuma, ${ }^{a}$ \\ Kentaro Yano, ${ }^{a}$ Hiroshi Arakawa, ${ }^{d}$ Kurt Hara,${ }^{b}$ Chihiro Miyajima, ${ }^{b}$ and Takuo Ogihara*, ${ }^{*, e}$ \\ ${ }^{a}$ Faculty of Pharmacy, Takasaki University of Health and Welfare; 60 Nakaorui-machi, Takasaki, Gunma 370-0033, \\ Japan: ${ }^{b}$ Kimica Corporation; 2-4-1 Yaesu, Chuo-ku, Tokyo 104-0028, Japan: ${ }^{c}$ Kendai Translational Research \\ Center; 60 Nakaorui-machi, Takasaki, Gunma 370-0033, Japan: ${ }^{d}$ Faculty of Pharmacy, Institute of Medical, \\ Pharmaceutical and Health Sciences, Kanazawa University; Kakuma-machi, Kanazawa, Ishikawa 920-1192, \\ Japan: and ${ }^{e}$ Laboratory of Clinical Pharmacokinetics, Graduate School of Pharmaceutical Sciences, Takasaki \\ University of Health and Welfare; 60 Nakaorui-machi, Takasaki, Gunma 370-0033, Japan. \\ Received July 12, 2018; accepted December 2, 2018
}

In this study, rats were fed a high-fat diet containing calcium alginate (Ca-Alg) for 5 weeks to examine the effects of Ca-Alg on lipid metabolism including triglyceride (TG) levels in the blood. We also investigated the mechanism of the TG-reducing effect of Alg in vitro. Rats were randomized into 5 groups: high-fat diet group $(14 \%(w / w)$ lard, HF); three Ca-Alg-containing diet groups $(2.5,5$ or $10 \%(w / w) ~ C a-A l g)$ and a resistant maltodextrin (RMD) diet group as a positive control (with $5 \%$ (w/w) RMD). The 10\% Ca-Alg group showed a significant reduction of body weight increase from the 7th day. In addition, the increase of TG in blood was significantly suppressed, and the amount of TG excreted in feces was increased. Increase of body fat mass was in the order HF $>$ RMD $>$ Ca-Alg $2.5 \%>$ Ca-Alg $5 \%>$ Ca-Alg $10 \%$, while the total weight of the extracted fat tissues was significantly reduced in the RMD, 5\% and $10 \%$ Ca-Alg groups. Hepatic pathology showed clear circular vacuoles apparently representing TG accumulation in the HF group, while fewer vacuoles were seen in the Ca-Alg groups. The results of in vitro experiments indicated that Ca-Alg does not directly inhibit lipase activity, but may suppress absorption of TG by forming non-absorbable macromolecular micelles containing TG. These results suggest that Ca-Alg promotes excretion and suppresses absorption of TG, leading to reduced blood TG levels, and decreased hepatic and total body accumulation of fat. The findings should be helpful for designing future clinical trials.

Key words calcium alginate; lipid; triglyceride; alginic acid; micelle; fat

\section{INTRODUCTION}

Heart disease and cerebrovascular disease, which are among the main causes of death in Japan, are frequently caused by damage to the blood vessels due to arteriosclerosis, i.e., thickening and loss of elasticity of the arterial walls. Thus, in order to prevent arteriosclerosis, it is necessary to control the accumulation of lipids and cholesterol (Cho) in the blood vessels. ${ }^{1-6)}$ Lipids in the body can be roughly classified as triglycerides (TG), Cho esters, free fatty acids, and phospholipids. Among them, fatty acids are utilized immediately for energy production, ${ }^{7)}$ while TG is stored in subcutaneous and internal organs and broken down into fatty acids as required. Excess lipid is stored in the form of TG. ${ }^{8)}$ Dyslipidemia, with increasing TG and Cho levels in the blood, leads to atherosclerosis (a form of arteriosclerosis involving buildup of fatty plaques in arteries), which in turn can lead to cardiovascular disease and stroke. Furthermore, accumulation of TG results in fatty liver, leading to decreased hepatic function, liver cirrhosis and potential morbidity, including myocardial infarction, cerebral infarction and angina pectoris, and eventually cancer. ${ }^{9-12)}$

Alginic acid (Alg) is a polysaccharide derived from brown seaweed. Sodium alginate (Na-Alg) is widely used not only as a food additive (as a thickening agent and stabilizer), but also as a health food to lower blood Cho level and inhibit weight gain. ${ }^{13-16)}$ Moreover, Na-Alg is used as a pharmaceutical, because of its protective effect on the gastric mucosa. Na-Alg also promotes the excretion of Cho in the human body. ${ }^{17}$ Consequently, Na-Alg is commercially available as a specific health food (Tokuho). ${ }^{18)}$ However, the sodium salt can potentially cause hypertension, which is a major risk factor for arteriosclerosis and dyslipidemia. ${ }^{19)}$ Therefore, we have focused on the effect of using the calcium salt of alginic acid (Ca-Alg).

Recently, we reported that $\mathrm{Ca}-\mathrm{Alg}$ reduces absorption and promotes excretion of both strontium ( $\mathrm{Sr}$ ) and cesium (Cs), whereas $\mathrm{Na}-\mathrm{Alg}$ is effective only for $\mathrm{Sr}^{20)}$ In addition, we investigated the relationship between the physical parameters of various metal ions and the binding affinity of these metal ions for Alg. ${ }^{21)}$ Moreover, we found that $\mathrm{Ca}-\mathrm{Alg}$ suppressed the elevation of blood glucose levels after ingestion of starch in rats and humans by inhibiting the degradation of maltose to glucose by $\alpha$-glucosidase. ${ }^{22,23)}$

Moreover, we reported the blood Cho-lowering effect of Ca$\mathrm{Alg}$ in rats. ${ }^{24)} \mathrm{Ca}-\mathrm{Alg}$ adsorbs bile acids in the intestine, inhibiting their reabsorption and enterohepatic circulation, and thus decreasing bile acid levels in the blood and enhancing their fecal excretion. Since bile acids are biosynthesized from Cho in the liver, it was considered that their biosynthesis is promoted, and this leads to the decrease in blood Cho level. ${ }^{25,26)}$ During that experiment, we found coincidentally that the TG 
level in blood was decreased. However, the mechanism of this TG-lowering effect was not established. Furthermore, it is necessary to examine the effect of $\mathrm{Ca}-\mathrm{Alg}$ in animals given a TG-loaded diet in place of Cho- and bile acid-loaded diets. In this study, we aimed to observe the inhibitory effect of Ca-Alg on elevated TG levels in the blood, hepatic and total body accumulation of fat, and body weight using rats fed a TG-loaded diet for 5 weeks. We also investigated the mechanism of the TG-reducing effect of Alg in vitro. These data are needed to provide a rational basis for designing a future clinical trial.

\section{MATERIALS AND METHODS}

Chemicals and Animals Lard, TG, Cho, sodium cholate, TG E-test WAKO, Cho E-test WAKO, total bile acid-test WAKO, 5-sulfosalicylic acid 2-hydrate, chloroform, sodium hydroxide, hydrochloric acid, potassium ferricyanide, allantoin, lecithin (from soybean) and gall powder were purchased from Wako Pure Chemical Industries, Ltd. (Osaka, Japan). Phenylhydrazine hydrochloride was purchased from SigmaAldrich Co. (MO, U.S.A.). High-molecular-weight Ca-Alg rich in guluronic acid was supplied by Kimica Corporation (Tokyo, Japan). Fibersol- $2^{\circledR}$, resistant maltodextrin (RMD), was purchased from Matsutani Chemical Industry Co., Ltd. (Hyogo, Japan). All other chemicals and solvents were analytical-grade commercial products.

Normal rat diet (AIN-93) was supplied by Clea Japan Inc. (Tokyo, Japan). ImpedoVET ${ }^{\circledR}$, a body composition meter for laboratory animals, was purchased from ImpediMed Ltd., (Brisbane, Australia). The animal study was performed in accordance with the Guidelines for the Care and Use of Laboratory Animals at Takasaki University of Health and Welfare and approved by the Committee of Ethics of Animal Experimentation of the University (approval No. 1505). Threeweek-old specific-pathogen-free male Sprague-Dawley (SD) rats were purchased from SLC Japan (Hamamatsu, Japan), and housed one animal per cage under controlled conditions (temperature of $24 \pm 1^{\circ} \mathrm{C}$, humidity of $50 \pm 10 \%$ ) with a consistent 12-h light/dark cycle, and free access to food and water.

In Vivo Experiments One week after purchase, rats weighing 92 to $106 \mathrm{~g}$ were randomized into 5 groups $(n=7$ each): a high-fat diet group (14\% (w/w) lard, HF); three CaAlg-containing diet groups $(14 \%(\mathrm{w} / \mathrm{w})$ lard, plus 2.5 or 5 or $10 \%(\mathrm{w} / \mathrm{w}) \mathrm{Ca}-\mathrm{Alg})$ and an RMD diet group $(14 \%(\mathrm{w} / \mathrm{w})$ lard, plus 5\% (w/w) RMD). RMD was selected as a positive control, because it is reported to increase TG excretion and reduce abdominal fat. ${ }^{27}$ Rats received the appropriate diet for 5 weeks. In a preliminary test conducted to set the dose of Ca-Alg, we confirmed that the cumulative blood TG amount (over 5 weeks) was significantly increased in the HF diet group compared with that in the normal diet group (Supplementary Fig. 1). Body weight and diet intake were measured and the general condition of the animals was monitored during this period. After 7, 14, 21, 28 and $35 \mathrm{~d}, 0.5 \mathrm{~mL}$ of blood was withdrawn from the jugular vein with a heparinized syringe under anesthesia with isofluran, and centrifuged at $1700 \times \boldsymbol{g}$ for $10 \mathrm{~min}$ to obtain plasma. The plasma concentration of TG was determined, and the amount of total body fat was measured using the body composition meter. Feces were collected during the final $3 \mathrm{~d}$ of the feeding period, dried in a lyophilizer, and then pulverized and heated in ethanol for two

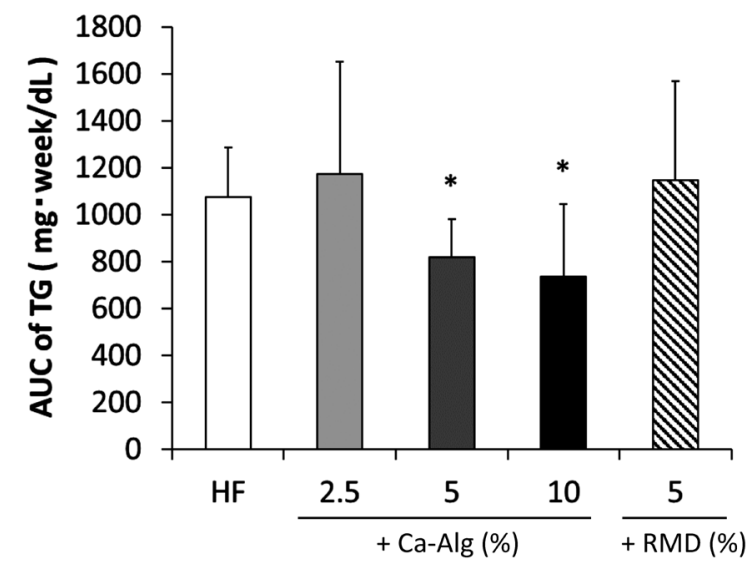

Fig. 1. Area under the Blood Concentration-Time Curve of TG in Rats after the Five-Week Feeding Period with High-Fat Diet or High-Fat Diet Containing Ca-Alg or High-Fat Diet Containing RMD

$$
(* p<0.05) \text {. }
$$

hours at $70^{\circ} \mathrm{C}$. The heating process was repeated four times, and $\mathrm{TG}$ and bile acids in the pooled extract were measured. After $35 \mathrm{~d}$, mesenteric adipose, epididymal adipose and retroperitoneal adipose tissues were removed and weighed. For safety monitoring, blood samples withdrawn after $35 \mathrm{~d}$ were used for the measurement of biochemical parameters, namely aspartate aminotransferase (AST), alanine aminotransferase (ALT), total Cho, phospholipid (PL), non-esterified fatty acid (NEFA), high-density lipoprotein (HDL), low-density lipoprotein (LDL), blood glucose (GLU), calcium (Ca), sodium $(\mathrm{Na})$, potassium $(\mathrm{K})$, and chloride $(\mathrm{Cl})$. After blood sampling, the animals were euthanized by exsanguination $(n=3$ each). Heart, liver, lung, kidneys, spleen, submandibular glands, parotid glands, mesenteric lymph nodes, stomach, intestines, testes, epididymis, thyroid glands, adrenal glands, white adipose tissue and brown adipose tissue were removed and immediately fixed in 10\% neutral buffered formalin. The tissues were immersed in paraffin, and sectioned at $4 \mu \mathrm{m}$ thickness. The slices were stained with hematoxylin and eosin, and examined microscopically. Moreover, liver sections of HF, RMD and $\mathrm{Ca}-\mathrm{Alg} 10 \%$ groups were stained with oil red $\mathrm{O}$.

In Vitro Experiments $\mathrm{Na}-\mathrm{Alg}$ was substituted for $\mathrm{Ca}-\mathrm{Alg}$ for the in vitro experiments, because Ca-Alg is insoluble in water. In order to measure lipase activity, Na-Alg $(0.3 \mathrm{~g})$ was suspended in $10 \mathrm{~mL}$ of water, and then diluted to give sample solutions containing 1,5 and $15 \mathrm{mg} / \mathrm{mL}$. Lipase-inhibitory activity was measured using a Lipase Kit S (DS Pharma Biomedical Co., Ltd., Osaka, Japan), with dimercaptopropanol tributyrate (BALB) as the substrate. The lipase solution contained $25 \mathrm{mU}$ porcine pancreatic lipase type II (Sigma-Aldrich) in citrate phosphate buffer ( $\mathrm{pH}$ 7.4). The reaction mixture, which consisted of $50 \mu \mathrm{L}$ substrate, $500 \mu \mathrm{L}$ chromogenic reagent, $50 \mu \mathrm{L} 0.5 \%$ lecithin and $0.2 \%$ gall solution, and $50 \mu \mathrm{L}$ sample solution, was preincubated at $30^{\circ} \mathrm{C}$ for $5 \mathrm{~min}$. Distilled water was used as a negative control and a lipase inhibitor, orlistat $(0.02 \mu \mathrm{g} / \mathrm{mL})$, was used as a positive control. After preincubation, $25 \mu \mathrm{L}$ lipase was added to the reaction mixtures, which were then incubated at $30^{\circ} \mathrm{C}$ for $30 \mathrm{~min}$ in the dark. The enzyme reaction was terminated by the addition of $1 \mathrm{~mL}$ stop reagent, and the absorbance at $412 \mathrm{~nm}$ was measured with a spectrophotometer, Sunrise Rainbow RC (Tecan Japan Co., Ltd., Kanagawa, Japan). A blank solution was prepared simi- 
larly, except that the stop reagent was added before addition of the substrate.

The lipase activity (\%) was calculated using the following formula:

[(sample absorbance) - (sample blank absorbance)]/

[(control absorbance $)-($ control blank absorbance $)] \times 100$

TG $(5 \mathrm{mg})$ was melted at $50^{\circ} \mathrm{C}$, and $3 \mathrm{~mL}$ of a lecithin and bile mixed solution (lecithin $0.5 \%$, bile powder $0.2 \%$ ) was added with stirring to prepare an emulsion. An aqueous solution of Na-Alg $(12 \mathrm{mg} / \mathrm{mL}, 3 \mathrm{~mL})$ or $3 \mathrm{~mL}$ of water was added to the prepared emulsion, and its state was observed.

Statistical Analysis Data are expressed as means standard deviation (S.D.). Statistical comparisons were made using Dunnett's test and the Wilcoxon test. Values of $p<0.05$ or 0.01 were considered significant.

\section{RESULTS}

Effect of Ca-Alg on Body Weight The increase of body weight was significantly suppressed from the 7 th day in the $10 \% \mathrm{Ca}-\mathrm{Alg}$ group. There was no significant difference in food intake or caloric intake among all groups (Table 1).

Effect of Ca-Alg on Lipid Metabolism TG concentration in the blood was significant suppressed by Ca-Alg 10\% (HF: $191 \pm 79$, + Ca-Alg 2.5\%: $193 \pm 73$, + Ca-Alg 5\%: $157 \pm 32$, + Ca-Alg 10\%: 102 \pm 30 , + RMD 5\%: $173 \pm 61 \mathrm{mg} / \mathrm{dL})$ at $7 \mathrm{~d}$, but not thereafter. In terms of the area under the blood concentration-time curve $(A U C)$ of TG concentration up to 5 weeks, a significant inhibitory effect on TG increase was observed in the $5 \%$ and $10 \% \mathrm{Ca}-\mathrm{Alg}$ groups (Fig. 1).

On the other hand, no significant differences were found in blood Cho levels within the study period (Supplementary Fig. 2a). In addition, Ca-Alg had no effect on the bile acid concentration in portal blood at $35 \mathrm{~d}$ (Supplementary Fig. 2b).

Effect of Ca-Alg on Fat During the test period, increase of body fat mass was in the order HF $>$ RMD $>$ Ca-Alg $2.5 \%$ $\geq \mathrm{Ca}-\mathrm{Alg} 5 \%>\mathrm{Ca}-\mathrm{Alg} 10 \%$. On $35 \mathrm{~d}$, the increase of body fat was significantly inhibited in the 5 and $10 \%$ Ca-Alg groups (Fig. 2). Based on the weight of adipose tissue excised at $35 \mathrm{~d}$, Ca-Alg and RMD had no effect on mesenteric fat (Fig. 3a). The increases of epididymal fat and retroperitoneal fat were significantly suppressed by Ca-Alg (5 and 10\%) and RMD (Figs. 3b, c). Total weight of the three excised visceral fat was significantly reduced in the RMD, 5\% and $10 \% \mathrm{Ca}-\mathrm{Alg}$ groups, compared with the control (Fig. 3d).

Excretion in Feces The total feces volume for the last $3 \mathrm{~d}$ during the test period was measured. A significant, dosedependent increase in fecal volume was observed in the CaAlg groups (Fig. 4a). The amount of TG per $1 \mathrm{~g}$ of feces was increased only in the RMD group (HF: $9.24 \pm 0.48,+\mathrm{Ca}-\mathrm{Alg}$ 2.5\%: $9.28 \pm 0.28$, + Ca-Alg 5\%: $9.54 \pm 0.38$, + Ca-Alg 10\%: $9.04 \pm 0.37$, + RMD 5\%: $9.69 \pm 0.32$ (mg/g feces). However, when the total excretion of TG was calculated from fecal TG

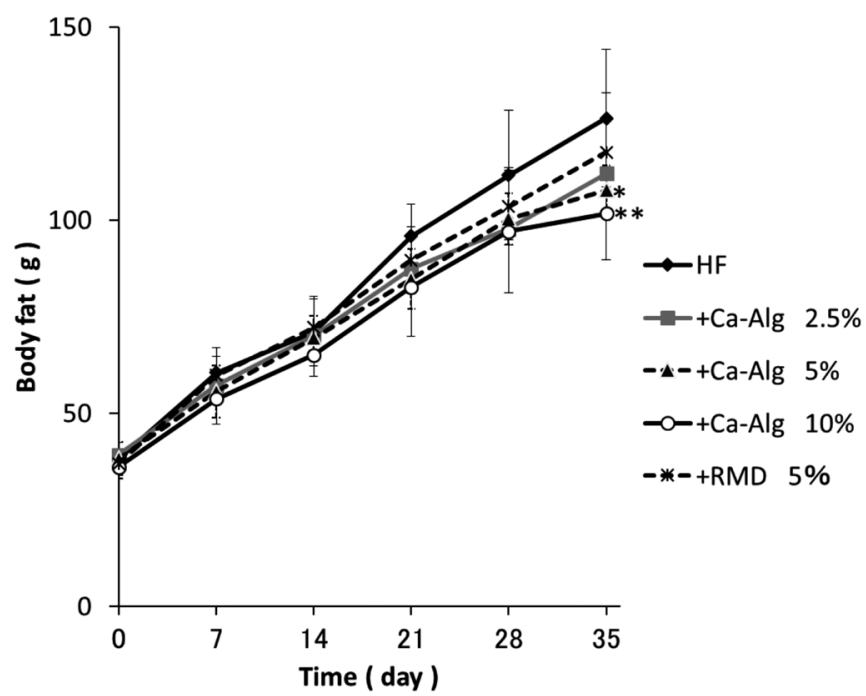

Fig. 2. Time-Course of Body Fat Increase during the Experimental Period

$$
\left({ }^{*} p<0.05, * * p<0.01\right) \text {. }
$$

Table 1. Cumulative Amount of Diet and Calorie Intake and Body Weight

\begin{tabular}{|c|c|c|c|c|c|c|}
\hline \multirow{2}{*}{ Parameter } & \multirow{2}{*}{ Time (d) } & \multirow{2}{*}{$\mathrm{HF}$} & \multicolumn{3}{|c|}{ + Ca-Alg (\%) } & \multirow{2}{*}{$\frac{+\mathrm{RMD}(\%)}{5}$} \\
\hline & & & 2.5 & 5 & 10 & \\
\hline \multirow[t]{5}{*}{ Cumulative amount of diet intake ( $\mathrm{g} /$ body) } & 7 & $92 \pm 6$ & $90 \pm 9$ & $93 \pm 5$ & $92 \pm 5$ & $88 \pm 4$ \\
\hline & 14 & $185 \pm 14$ & $179 \pm 18$ & $188 \pm 11$ & $183 \pm 10$ & $179 \pm 8$ \\
\hline & 21 & $282 \pm 27$ & $275 \pm 31$ & $288 \pm 17$ & $283 \pm 21$ & $275 \pm 15$ \\
\hline & 28 & $392 \pm 41$ & $382 \pm 47$ & $402 \pm 25$ & $393 \pm 36$ & $385 \pm 23$ \\
\hline & 35 & $507 \pm 59$ & $496 \pm 57$ & $514 \pm 32$ & $504 \pm 50$ & $495 \pm 32$ \\
\hline \multirow[t]{5}{*}{ Cumulative amount of calorie intake (kcal/body) } & 7 & $413 \pm 29$ & $399 \pm 39$ & $401 \pm 21$ & $382 \pm 19$ & $380 \pm 17$ \\
\hline & 14 & $831 \pm 64$ & $787 \pm 81$ & $810 \pm 48$ & $757 \pm 40$ & $771 \pm 33$ \\
\hline & 21 & $1270 \pm 121$ & $1213 \pm 135$ & $1242 \pm 75$ & $1169 \pm 88$ & $1187 \pm 64$ \\
\hline & 28 & $1766 \pm 183$ & $1683 \pm 209$ & $1733 \pm 108$ & $1624 \pm 149$ & $1662 \pm 100$ \\
\hline & 35 & $2280 \pm 266$ & $2184 \pm 249$ & $2220 \pm 137$ & $2082 \pm 206$ & $2134 \pm 137$ \\
\hline \multirow[t]{6}{*}{ Body weight (g) } & 0 & $99 \pm 4$ & $98 \pm 5$ & $98 \pm 4$ & $98 \pm 4$ & $98 \pm 5$ \\
\hline & 7 & $148 \pm 8$ & $143 \pm 12$ & $143 \pm 5$ & $137 \pm 7^{*}$ & $142 \pm 7$ \\
\hline & 14 & $189 \pm 13$ & $180 \pm 17$ & $183 \pm 9$ & $170 \pm 11 *$ & $182 \pm 9$ \\
\hline & 21 & $238 \pm 18$ & $228 \pm 23$ & $227 \pm 14$ & $212 \pm 17^{*}$ & $231 \pm 14$ \\
\hline & 28 & $285 \pm 23$ & $269 \pm 29$ & $273 \pm 18$ & $253 \pm 25^{*}$ & $273 \pm 19$ \\
\hline & 35 & $323 \pm 33$ & $304 \pm 30$ & $305 \pm 20$ & $286 \pm 32 *$ & $305 \pm 20$ \\
\hline
\end{tabular}

The data are mean \pm S.D. $(n=7)$. The significance of differences between HF group and the Ca-Alg containing groups or the RMD group was determined by means of Dunnett's test $(* p<0.05)$. 

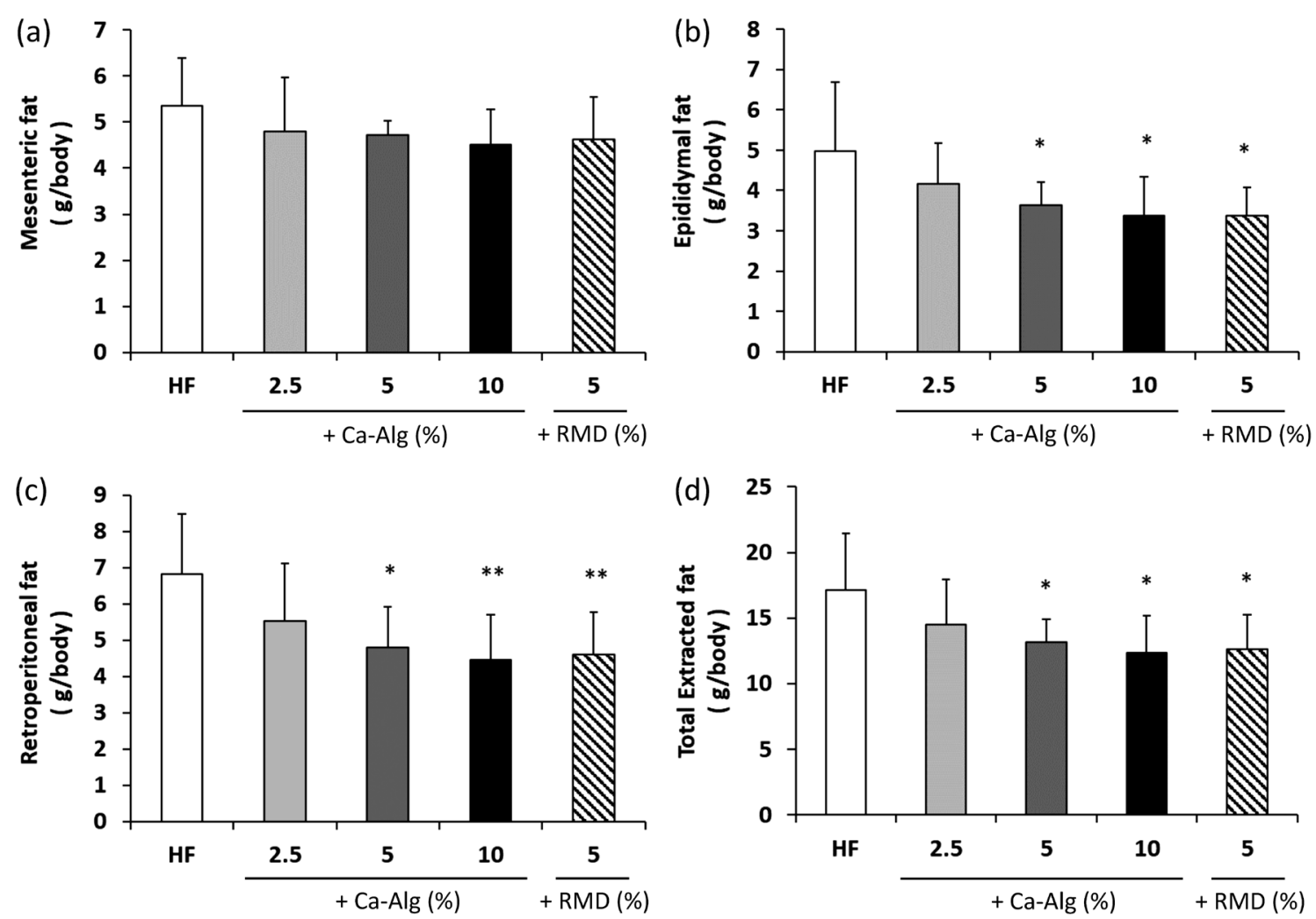

Fig. 3. Weight of Fat Tissues after the Five-Week Feeding Period with High-Fat Diet or High-Fat Diet Containing Ca-Alg or High-Fat Diet Containing RMD

(a) Mesenteric fat, (b) epididymal fat, (c) retroperitoneal fat, (d) total fat ((a) + (b) + (c)). $\left({ }^{*} p<0.05, * * p<0.01\right)$.

(a)
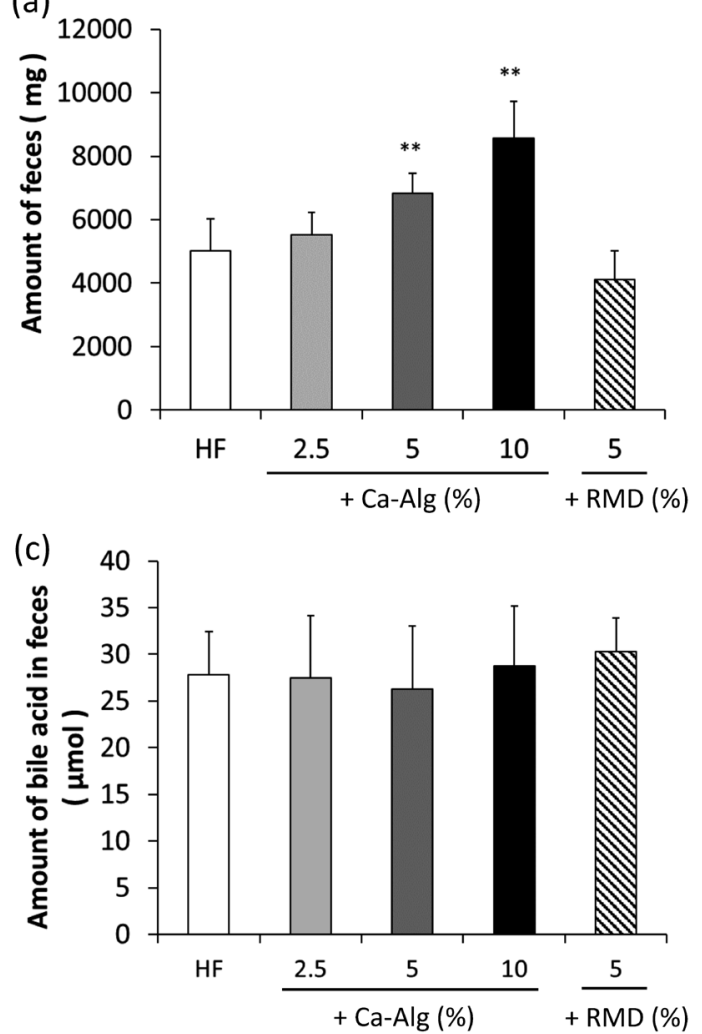

(b)

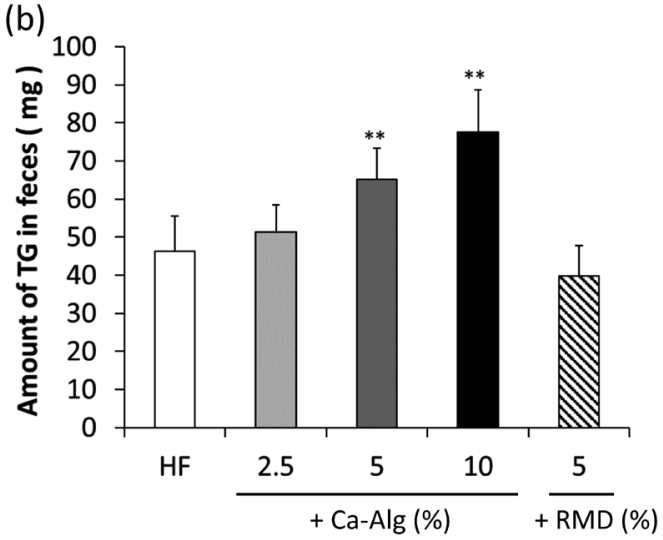

Fig. 4. (a) Amount of Feces Produced by Rats after the Five-Week Feeding Period with High-Fat Diet or High-Fat Diet Containing Ca-Alg or HighFat Diet Containing RMD; (b) Amount of TG in Rat Feces after the Five-Week Feeding Period with High-Fat Diet Or High-Fat Diet Containing CaAlg or High-Fat Diet Containing RMD; (c) Amount of Bile Acid in rat Feces after the Five-Week Feeding Period with High-Fat Diet or High-Fat Diet Containing Ca-Alg or High-Fat Diet Containing RMD 
(a)

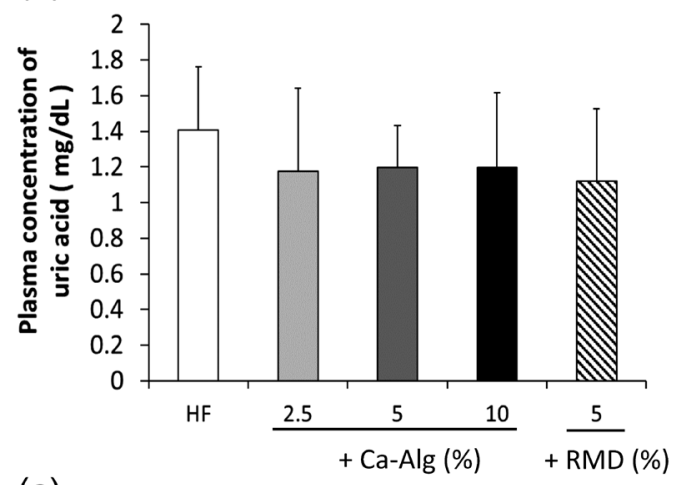

(c)

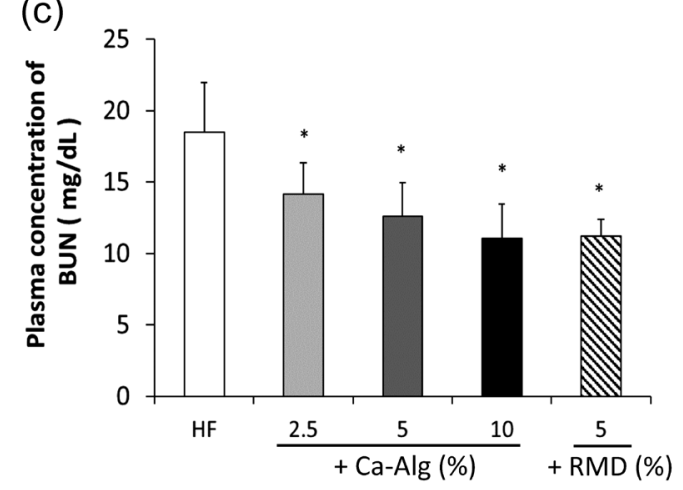

(b)

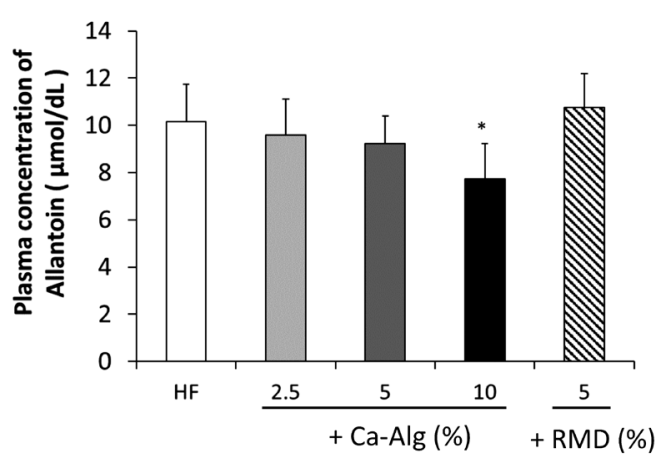

Fig. 5. Plasma Concentrations of (a) Uric Acid, (b) Allantoin and (c) BUN in Rats after the Five-Week Feeding Period with High-Fat Diet or HighFat Diet Containing Ca-Alg or High-Fat Diet Containing RMD

$(* p<0.05)$.

Table 2. Liver Weight and Biochemical Parameters in Rats (at Day 35)

\begin{tabular}{|c|c|c|c|c|c|}
\hline \multirow{2}{*}{ Parameters } & \multirow{2}{*}{$\mathrm{HF}$} & \multicolumn{3}{|c|}{ + Ca-Alg (\%) } & \multirow{2}{*}{$\frac{+\mathrm{RMD}(\%)}{5}$} \\
\hline & & 2.5 & 5 & 10 & \\
\hline Liver weight (mg) & $11.0 \pm 0.3$ & $12.7 \pm 1.6$ & $11.4 \pm 2.4$ & $10.4 \pm 2.2$ & $12.6 \pm 2.0$ \\
\hline LDLC (mg/dL) & $9 \pm 2$ & $8 \pm 1$ & $8 \pm 1$ & $9 \pm 1$ & $9 \pm 2$ \\
\hline HDLC (mg/dL) & $32 \pm 4$ & $30 \pm 2$ & $28 \pm 3$ & $28 \pm 2$ & $31 \pm 4$ \\
\hline PL (mg/dL) & $139 \pm 25$ & $130 \pm 12$ & $112 \pm 7 * *$ & $119 \pm 5^{*}$ & $128 \pm 13$ \\
\hline NEFA $(\mu \mathrm{Eq} / \mathrm{L})$ & $1003 \pm 380$ & $1134 \pm 837$ & $634 \pm 179$ & $641 \pm 254$ & $959 \pm 409$ \\
\hline $\mathrm{Na}(\mathrm{mmol} / \mathrm{L})$ & $142 \pm 1$ & $142 \pm 2$ & $142 \pm 2$ & $142 \pm 1$ & $142 \pm 1$ \\
\hline $\mathrm{K}(\mathrm{mmol} / \mathrm{L})$ & $5.4 \pm 0.4$ & $5.3 \pm 0.5$ & $5.1 \pm 0.5$ & $5.3 \pm 0.7$ & $5.1 \pm 0.7$ \\
\hline $\mathrm{Ca}(\mathrm{mmol} / \mathrm{L})$ & $10.7 \pm 0.2$ & $10.7 \pm 0.3$ & $10.3 \pm 0.3$ & $10.7 \pm 0.3$ & $10.6 \pm 0.4$ \\
\hline $\mathrm{Cl}(\mathrm{mmol} / \mathrm{L})$ & $101 \pm 2$ & $101 \pm 4$ & $102 \pm 1$ & $103 \pm 1$ & $102 \pm 2$ \\
\hline Cre $(\mathrm{mg} / \mathrm{dL})$ & $0.42 \pm 0.06$ & $0.37 \pm 0.08$ & $0.38 \pm 0.05$ & $0.37 \pm 0.05$ & $0.35 \pm 0.04^{*}$ \\
\hline ALP (U/L) & $1411 \pm 423$ & $1278 \pm 335$ & $1831 \pm 1236$ & $1352 \pm 353$ & $1293 \pm 171$ \\
\hline GLU (mg/dL) & $223 \pm 39$ & $220 \pm 26$ & $229 \pm 28$ & $224 \pm 15$ & $213 \pm 34$ \\
\hline ALT (U/L) & $38 \pm 8$ & $31 \pm 7$ & $32 \pm 12$ & $29 \pm 7$ & $36 \pm 8$ \\
\hline AST (U/L) & $88 \pm 15$ & $82 \pm 19$ & $94 \pm 38$ & $81 \pm 17$ & $72 \pm 10$ \\
\hline
\end{tabular}

The data are mean \pm S.D. $(n=7)$. The significance of differences between the HF group and the Ca-Alg containing groups or the RMD group was determined by means of Dunnett's test $(* p<0.05, * * p<0.01)$.

concentration and fecal volume, a significant, concentrationdependent increase in fecal excretion of TG was found in the Ca-Alg groups (Fig. 4b). Ca-Alg did not affect bile acid excretion in feces (Fig. 4c).

Effect of Ca-Alg on Nitrogen Metabolism The concentrations of uric acid, allantoin and BUN in plasma on $35 \mathrm{~d}$ were measured. There was a tendency for decreased uric acid concentration, and significant, dose-dependent decreases of allantoin and BUN concentration were observed in the $\mathrm{Ca}-\mathrm{Alg}$ groups (Fig. 5).
Liver Weight and Biochemical Parameters There was no significant difference in liver weight. Several changes were observed in the biochemical parameters, but they remained within the physiological ranges, and there appeared to be no safety issue (Table 2).

Pathological Assessment Vesicles considered to represent accumulation of TG in the liver were observed in all groups. In addition, oil red $\mathrm{O}$ staining of liver sections from the HF, RMD and Ca-Alg 10\% groups was performed, and it was confirmed that the vesicles were lipid droplets (Fig. 6). The 

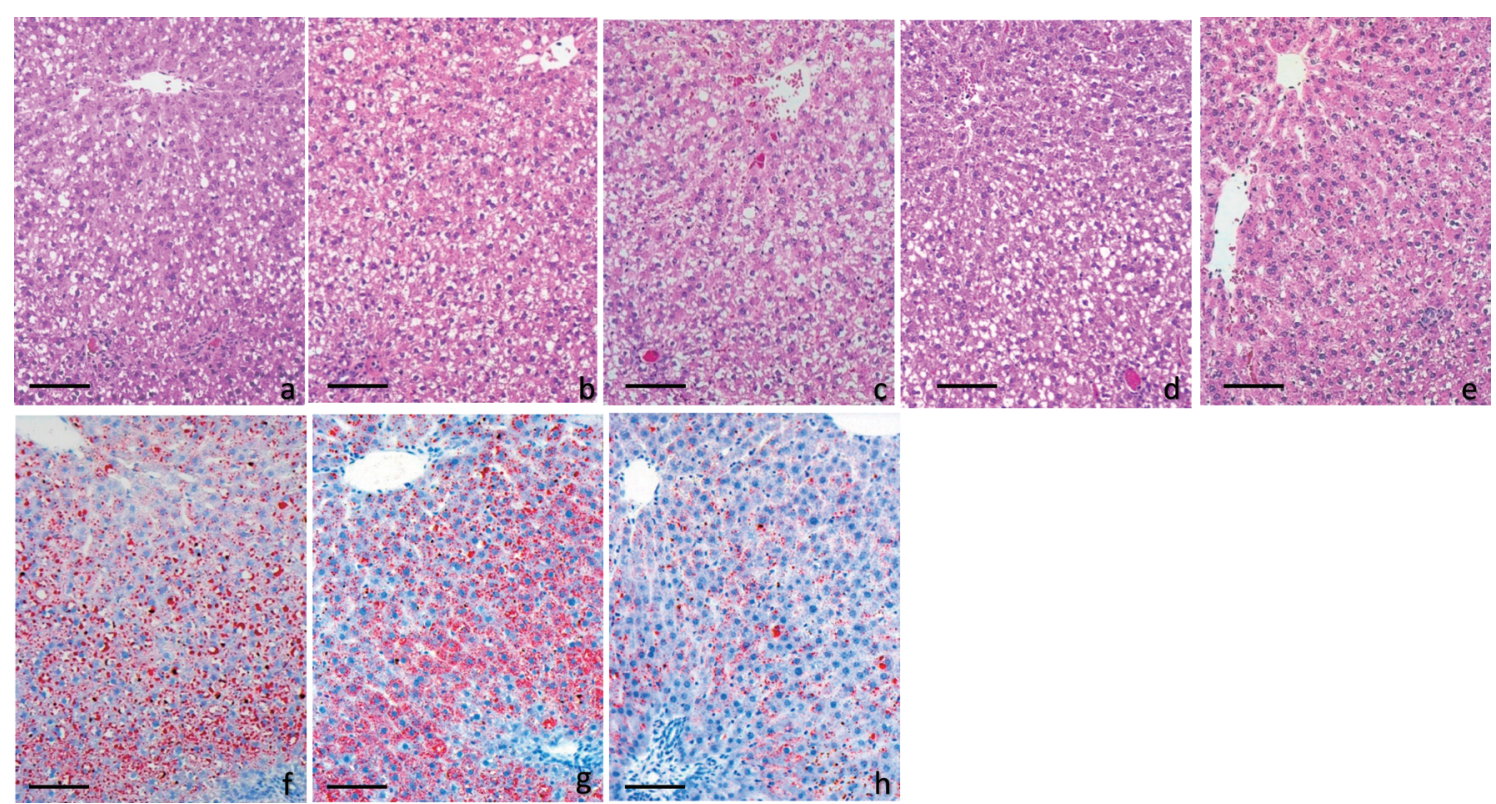

Fig. 6. Histopathology of Hepatocytes from Rats Given High-Fat Diet (a and f), High-Fat Diet Containing RMD (b and g), High-Fat Diet Containing 2.5\% Ca-Alg (c), High-Fat Diet Containing 5\% Ca-Alg (d) and High-Fat Diet Containing 10\% Ca-Alg (e and h)

The results of hematoxylin and eosin staining ( $, \mathrm{b}, \mathrm{c}, \mathrm{d}$ and e) and oil red $\mathrm{O}$ staining ( $\mathrm{f}, \mathrm{g}$ and $\mathrm{h}$ ) show that the decrease of vacuoles in hematoxylin and eosin staining is consistent with the decrease of lipids in hepatocytes. Bar: $100 \mu \mathrm{m}$.

Table 3. Histological Findings in Rat Liver, Kidney, Pancreas, Mesenteric Adipose Tissue and Adipose Tissue around Testis after Five-Week Feeding Period

\begin{tabular}{|c|c|c|c|c|c|c|c|c|c|c|c|c|c|c|c|}
\hline \multirow{2}{*}{ Tissue findings } & \multirow{2}{*}{\multicolumn{3}{|c|}{ Treatment HF }} & \multicolumn{9}{|c|}{$+\mathrm{Ca}-\mathrm{Alg}(\%)$} & \multicolumn{3}{|c|}{$+\mathrm{RMD}(\%)$} \\
\hline & & & & \multicolumn{3}{|c|}{2.5} & \multicolumn{3}{|c|}{5} & \multicolumn{3}{|c|}{10} & \multicolumn{3}{|c|}{5} \\
\hline \multicolumn{16}{|l|}{ Liver } \\
\hline Vacuoles in hepatocytes in peripheral area & \pm & + & + & + & + & + & + & + & \pm & \pm & \pm & \pm & \pm & + & + \\
\hline \multicolumn{16}{|l|}{ Kidney } \\
\hline Small granuloma & - & - & - & - & - & - & - & - & - & \pm & - & - & - & - & - \\
\hline Increase in basophilic tubules & - & - & - & - & - & - & - & - & - & - & - & - & - & - & \pm \\
\hline Pancreas & NAD & NAD & NAD & NAD & NAD & NAD & NAD & NAD & NAD & NAD & NAD & NAD & NAD & NAD & NAD \\
\hline Mesenteric adipose tissue & - & - & - & - & - & - & - & - & - & - & - & - & - & - & - \\
\hline Lymphocytic infiltration & - & \pm & \pm & - & - & \pm & & + & \pm & - & \pm & \pm & \pm & + & - \\
\hline Adipose tissue around testis & NAD & NAD & NAD & NAD & NAD & NAD & NAD & NAD & NAD & NAD & NAD & NAD & NAD & NAD & NAD \\
\hline
\end{tabular}

Grade of changes; - : within normal limit, \pm : slight, + : moderate. NAD; no abnormalities detected.

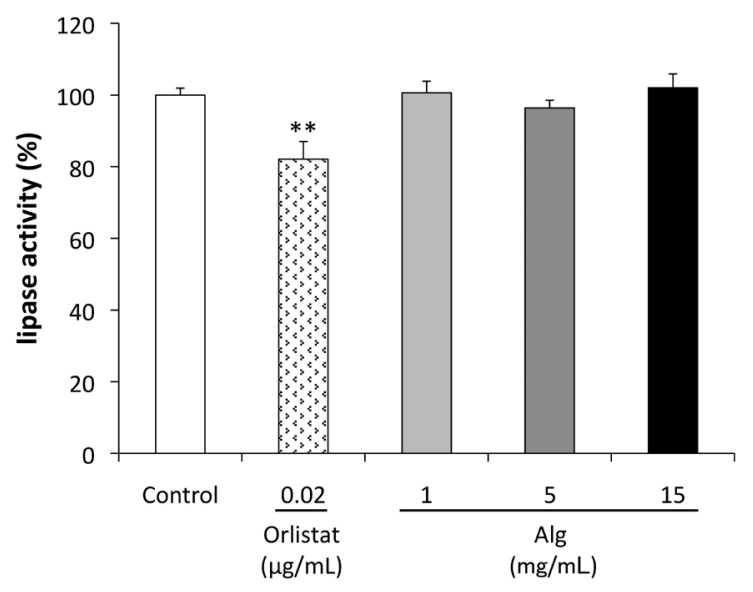

Fig. 7. Inhibitory Effect of Alg on Lipase Activity $n=3, * * p<0.01$.

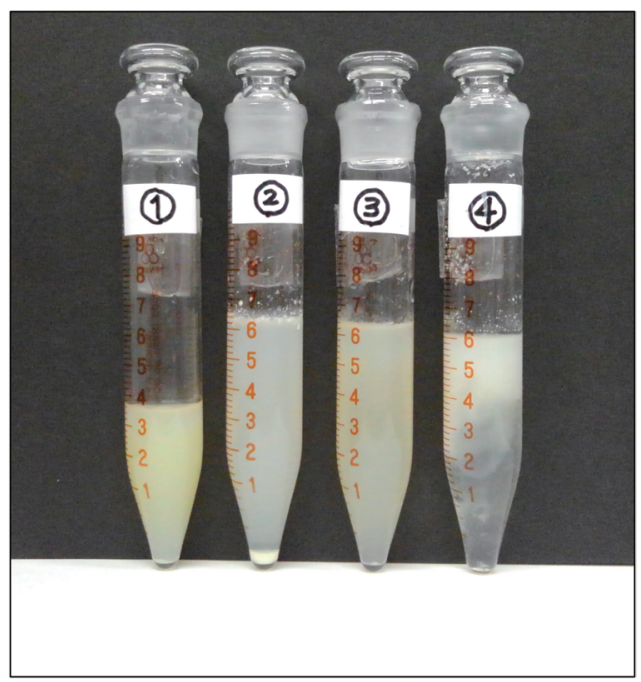

Fig. 8. Effect of Na-Alg on Micelle Stability

(1) TG + lecithin + gall, (2) TG + lecithin + gall + water, (3) TG + lecithin + gall + $\mathrm{Na}-\mathrm{Alg}$, (4) TG + lecithin + gall + Na-Alg (after $5 \mathrm{~d}$ ). 
amount of vesicles was decreased in the $10 \% \mathrm{Ca}$-Alg group compared with the HF and RMD group. The oil red $\mathrm{O}$ staining data supported this conclusion. No differences were observed in other organs and tissues among all groups (Table 3).

Inhibitory Effect on Lipase Activity in Vitro No decrease in lipase activity by Na-Alg was observed. On the other hand, lipase activity was decreased significantly to $82.1 \%$ by orlistat, used as a positive control (Fig. 7).

Effect on Micelle Stability in Vitro When Na-Alg was added to an emulsion composed of TG, bile and lecithin, the emulsion was maintained, and a creaming phenomenon was confirmed after $5 \mathrm{~d}$. When water was added to the emulsion, it disintegrated, exhibiting lecithin precipitation, and release of TG on the liquid surface (Fig. 8).

\section{DISCUSSION}

The blood Cho-lowering effect of Ca-Alg and its mechanism in rats are well established. ${ }^{24)}$ In the present study, we focused on the effects of Ca-Alg on TG and body fat weight in rats fed a fat-loaded diet. The results indicate that $\mathrm{Ca}-\mathrm{Alg}$ significantly decreases the AUC of blood TG concentration. At the same time, the amount of feces and the amount of TG excreted in feces increased, though bile acid excretion in feces and blood Cho were unaffected. Therefore, it appears that blood TG is lowered through direct suppression of TG absorption, independently of Cho.

The in vitro results suggested that Alg did not directly inhibit lipase activity, but stabilized bile acid micelles containing TG. It is known that Na-Alg reduces the Cho level in blood. ${ }^{14,18)}$ Moreover, we reported that $\mathrm{Ca}-\mathrm{Alg}$ promoted bile acid excretion, inducing catabolism from cholesterol to bile acid. ${ }^{24)}$ Therefore, it seems likely that $\mathrm{Ca}-\mathrm{Alg}$ adsorbed TG-containing bile-acid micelles, forming large micelles that would not be absorbed.

Increase of mesenteric fat was not suppressed in any of the groups, though the increases of epididymal fat and retroperitoneal fat were suppressed in the $5 \%$ and $10 \%$ Ca-Alg groups, and the RMD group. Significant suppression of total body fat mass was observed only in the Ca-Alg groups. To assess these effects, we defined $\Delta \mathrm{HF}$ as the difference of total body fat mass between an individual group and the HF group, and $\Delta \mathrm{HF}^{\prime}$ as the difference in total weight of mesenteric, epididymal and retroperitoneal fats between an individual group and the HF group. The ratio of $\Delta \mathrm{HF}$ to $\Delta \mathrm{HF}^{\prime}\left(\Delta \mathrm{HF} / \Delta \mathrm{HF}^{\prime}\right)$ was about 5 in the Ca-Alg groups and about 2 in the RMD group (Table 4). These results suggest that $\mathrm{Ca}-\mathrm{Alg}$ mainly suppresses the increase of non-visceral fat, and is more potent than RMD in this respect. In addition, the ease of fat reduction by $\mathrm{Ca}-\mathrm{Alg}$ and RMD is in the order of: epididymal fat and retroperitoneal fat $>$ subcutaneous fat (non-visceral fat) $>$ mesenteric fat.

As regards the mechanism of $\mathrm{Ca}-\mathrm{Alg}$ action, hepatic pathology showed that clear circular fatty droplets presumed to represent TG accumulation were present in the HF group, but were reduced in the $10 \% \mathrm{Ca}-\mathrm{Alg}$ group. Therefore, Ca-Alg may promote excretion and suppress absorption of TG, leading to inhibition of its hepatic accumulation.

Because a correlation between visceral fat mass and uric acid concentration in blood has been reported, ${ }^{28,29)}$ we measured uric acid, allantoin and blood urea nitrogen (BUN) in plasma. Allantoin and BUN showed significant dose-dependent decreases in the Ca-Alg groups. However, it is not clear whether this result is specific to a high fat diet-fed condition, and it will be necessary to investigate whether Ca-Alg influences uric acid metabolism in normally fed animals without fat accumulation.

No significant differences in liver weight were found among the groups, suggesting that Ca-Alg shows little or no hepatotoxicity. Several changes were observed in biochemical parameters, but all of them remained within the physiological ranges, suggesting that $\mathrm{Ca}-\mathrm{Alg}$ is safe at the levels examined.

In a previous study, ${ }^{30)}$ it was found that RMD inhibited TG elevation in the blood after a single administration. However, in our study, steady-state blood TG levels were not decreased in rats fed a RMD mixed diet for 5 weeks. Therefore, it appears that $\mathrm{Ca}-\mathrm{Alg}$ reduces the concentration of TG level in the blood in the steady state, whereas the effect of RMD might be temporary. Moreover, we found that the amount of TG excreted in feces did not increase in the RMD group, in contrast to the previous result. ${ }^{27)}$ The fecal TG concentration in the HF group was similar in both studies, but the fecal weight was increased only in the previous study. Although the reason for the difference is unclear, it might be related to the different feed composition. Overall, we consider that the TG-reducing effect of RMD might be limited in the steady state.

We previously reported that the amount of fecal excretion of bile acids was increased when Cho- and bile acid-loaded diets were given to rats, ${ }^{24)}$ whereas the excretion of fecal bile acid was not increased in the present study. Bile acids are essential for life, and we think Ca-Alg may exert its excretionpromoting effect only when they are present in excess, but not when bile acid concentrations are within the physiological range.

In conclusion, our results show that $\mathrm{Ca}-\mathrm{Alg}$ suppresses the increase in blood TG and body fat in rats fed a high-fat diet. The in vitro results suggest that Alg suppressed the absorp-

Table 4. Comparison of Decreases of Body Fat Mass and Extracted Fat Amount

\begin{tabular}{|c|c|c|c|c|c|c|}
\hline & & \multicolumn{2}{|c|}{ Body fat } & \multicolumn{2}{|c|}{ Extracted fat } & \multirow[b]{2}{*}{$\Delta \mathrm{HF} / \Delta \mathrm{HF}$} \\
\hline & & Body fat (g) & $\Delta \mathrm{HF}$ & Total fat ${ }^{\dagger}(\mathrm{g})$ & $\Delta \mathrm{HF}^{\prime}$ & \\
\hline $\mathrm{HF}$ & & $126.4 \pm 17.8$ & - & $17.1 \pm 4.3$ & - & - \\
\hline \multirow[t]{3}{*}{$+\mathrm{Ca}-\mathrm{Alg}(\%)$} & 2.5 & $112.1 \pm 18.5$ & -14.4 & $14.5 \pm 3.4$ & -2.6 & 5.4 \\
\hline & 5 & $107.8 \pm 6.4^{*}$ & -18.6 & $13.2 \pm 1.7^{*}$ & -4.0 & 4.7 \\
\hline & 10 & $101.8 \pm 12.0 * *$ & -24.7 & $12.4 \pm 2.8 *$ & -4.8 & 5.1 \\
\hline + RMD (\%) & 5 & $117.7 \pm 15.4$ & -8.8 & $12.6 \pm 2.7^{*}$ & -4.6 & 1.9 \\
\hline
\end{tabular}

${ }^{\dagger}$ Total fat is summation of mesenteric fat, epididymal fat and retroperitoneal fat. The significance of differences between the HF group and the Ca-Alg containing groups or the RMD group was determined by means of Dunnett's test $(* p<0.05, * * p<0.01)$. 
tion of TG by forming macromolecular micelles containing TG, and/or may protect TG from attack by lipases. Overall, our results suggest that $\mathrm{Ca}-\mathrm{Alg}$ promotes excretion and suppresses absorption of TG, leading to reduced blood TG levels, and decreased hepatic and total body accumulation of TG. The findings should be helpful for designing future clinical trials.

Conflict of Interest Chihiro Miyajima, Kurt Hara and Fumiyoshi Kasahara are employees of Kimica Corporation. The other authors have no conflict of interest.

Supplementary Materials The online version of this article contains supplementary materials.

\section{REFERENCES}

1) "Analysis by cause of death": 〈https://www.mhlw.go.jp/english/ database/db-hw/lifetb16/d1/lifetb16-04.pdf), Ministry of Health, Labour and Welfare, 2016.

2) Kitamura $A$, Iso $H$, Naito $Y$, Iida $M$, Konishi $M$, Folsom $A R$, Sato S, Kiyama M, Nakamura M, Sankai T, Shimamoto T, Komachi Y. High-density lipoprotein cholesterol and premature coronary heart disease in urban Japanese men. Circulation, 89, 2533-2539 (1994).

3) Iso $H$, Naito $Y$, Sato $S$, Kitamura A, Okamura T, Sankai T, Shimamoto $\mathrm{T}$, Iida $\mathrm{M}$, Komachi $\mathrm{Y}$. Serum triglycerides and risk of coronary heart disease among Japanese men and women. Am. J. Epidemiol., 153, 490-499 (2001).

4) NIPPON DATA80 Research Group. Impact of elevated blood pressure on mortality from all causes, cardiovascular diseases, heart disease and stroke among Japanese: 14 year follow-up of randomly selected population from Japanese (Nippon Data 80). J. Hum. Hypertens., 17, 851-857 (2003).

5) Teramoto T. Dietary management in Japan Atherosclerosis Society (JAS) guidelines for the Prevention of atherosclerotic cardiovascular diseases in Japanese-2012 Version-. The Japanese Journal of Nutrition and Dietetics, 71, 3-13 (2013).

6) Abifadel M, Varret M, Rabès JP, Allard D, Ouguerram K, Devillers M, Cruaud C, Benjannet S, Wickham L, Erlich D, Derré A, Villéger L, Farnier M, Beucler I, Bruckert E, Chambaz J, Chanu B, Lecerf JM, Luc G, Moulin P, Weissenbach J, Prat A, Krempf M, Junien C, Seidah NG, Boileau C. Mutations in PCSK9 cause autosomal dominant hypercholesterolemia. Nat. Genet., 34, 154-156 (2003).

7) Yamada K. Fat palatability and lipid metabolism in human body. Bulletin of School of Health Sciences Sapporo Medical University, 11, 1-10 (2008).

8) Shibata S, Ikeda Y. Chrono-nutrition of macro-nutrition including lipids. Journal of Lipid Nutrition, 24, 53-60 (2015).

9) Koda M, Kawakami M, Murawaki Y, Senda M. The impact of visceral fat in nonalcoholic fatty liver disease: cross-sectional and longitudinal studies. J. Gastroenterol., 42, 897-903 (2007).

10) Vega GL, Chandalia M, Szczepaniak LS, Grundy SM. Metabolic correlates of nonalcoholic fatty liver in women and men. Hepatology, 46, 716-722 (2007).

11) Jakobsen MU, Berentzen T, Sorensen TI, Overvad K. Abdominal obesity and fatty liver. Epidemiol. Rev., 29, 77-87 (2007).

12) Eguchi Y, Eguchi T, Mizuta T, Ide Y, Yasutake T, Iwakiri R, Hisatomi A, Ozaki I, Yamamoto K, Kitajima Y, Kawaguchi Y, Kuroki $\mathrm{S}$, Ono N. Visceral fat accumulation and insulin resistance are important factors in nonalcoholic fatty liver disease. J. Gastroenterol., 41, 462-469 (2006).

13) Kimica Corporation Web. «https://www.kimica.jp/en/alginate/s, 2019.

14) Nishizawa M, Kuda T, Yamagishi T, Tsuji K. Effect of depolymerized sodium alginate on the excretion of cholesterol from rats. $J$.
Home Econ. Jpn, 48, 689-693 (1997).

15) Peters HP, Koppert RJ, Boers HM, Ström A, Melnikov SM, Haddeman E, Schuring EA, Mela DJ, Wiseman SA. Dose-dependent suppression of hunger by a specific alginate in a low-viscosity drink formulation. Obesity, 19, 1171-1176 (2011).

16) Georg Jensen M, Kristensen M, Astrup A. Effect of alginate supplementation on weight loss in obese subjects completing a 12-week energy-restricted diet: a randomized controlled trial. Am. J. Clin. Nutr., 96, 5-13 (2012).

17) Pharmaceuticals and Medical Devices Agency Web. 〈http://www. info.pmda.go.jp/downfiles/ph/PDF/200343_2329116S1094_2_01.pdf), 2013.

18) Asaoka T, Iwatuka H, Minowa H. Effect of "CHOLECUT", a drink containing depolymerized sodium alginate, on serum total cholesterol levels in healthy mates. Japanese Journal of Nutritional Assessment, 13, 460-464 (1996).

19) Fujita T, Henry WL, Bartter FC, Lake CR, Delea CS. Factors influencing blood pressure in salt-sensitive patients with hypertension. Am. J. Med., 69, 334-344 (1980).

20) Idota $\mathrm{Y}$, Harada H, Tomono T, Morimoto K, Kobayashi S, Kakinuma C, Miyajima C, Kasahara F, Ogihara T. Alginate enhances excretion and reduces absorption of strontium and cesium in rats. Biol. Pharm. Bull., 36, 485-491 (2013).

21) Idota Y, Kogure Y, Kato T, Yano K, Arakawa H, Miyajima C, Kasahara F, Ogihara T. Relationship between physical parameters of various metal ions and binding affinity for alginate. Biol. Pharm. Bull., 39, 1893-1896 (2016).

22) Idota $Y$, Kato $T$, Shiragami $K$, Koike M, Yokoyama A, Takahashi $H$, Yano K, Ogihara T. Mechanism of suppression of blood glucose level by calcium alginate in rats. Biol. Pharm. Bull., 41, 1362-1366 (2018).

23) Kato T, Idota Y, Shiragami K, Koike M, Nishibori F, Tomokane M, Seki T, Itabashi K, Hakoda K, Takahashi H, Yano K, Kobayashi T, Obara N, Ogihara T. Randomized, double-blind, crossover clinical trial of the effect of calcium alginate in noodles on postprandial blood glucose level. Biol. Pharm. Bull., 41, 1367-1371 (2018).

24) Idota Y, Kogure Y, Kato T, Ogawa M, Kobayashi S, Kakinuma C, Yano K, Arakawa H, Miyajima C, Kasahara F, Ogihara T. Cholesterol-lowering effect of calcium alginate in rats. Biol. Pharm. Bull., 39, 62-67 (2016).

25) Peters AM, Walters JR. Recycling rate of bile acids in the enterohepatic recirculation as a major determinant of whole body 75SeHCAT retention. Eur. J. Nucl. Med. Mol. Imaging, 40, 1618-1621 (2013).

26) Galatola G, Jazrawi RP, Bridges C, Joseph AE, Northfield TC Direct measurement of first-pass ileal clearance of a bile acid in humans. Gastroenterology, 100, 1100-1105 (1991).

27) Kishimoto $Y$, Yoshikawa Y, Miyazato $\mathrm{S}, \mathrm{Oga} H$, Yamada T, Tagami $\mathrm{H}$, Hashizume C, Yamamoto K. Effect of resistant mal to dextrin on digestion and absorption of lipids. J. Health Sci., 55, 838-844 (2009).

28) Tamba $S$, Nishizawa H, Funahashi $T$, Okauchi $Y$, Ogawa $T$, Noguchi M, Fujita K, Ryo M, Kihara S, Iwahashi H, Yamagata K, Nakamura T, Shimomura I, Matsuzawa Y. Relationship between the serum uric acid level, visceral fat accumulation and serum adiponectin concentration in Japanese men. Intern. Med., 47, 1175-1180 (2008).

29) Sakamaki K, Maejima Y, Tokita Y, Masamura Y, Kumamoto K, Akuzawa M, Nagano N, Nakajima K, Shimomura K, Takenoshita $\mathrm{S}$, Shimomura Y. Impact of the visceral fat area measured by dual impedance method on the diagnostic components of metabolic diseases in a middle-aged Japanese population. Intern. Med., 55, 1691-1696 (2016)

30) Kishimoto Y, Oga H, Tagami H, Okuma K, Gordon DT. Suppressive effect of resistant maltodextrin on postprandial blood triacylglycerol elevation. Eur. J. Nutr., 46, 133-138 (2007). 